\title{
NSPWMLFMA: A Low Frequency Stable Formulation of the MLFMA in Three Dimensions
}

\author{
I. Bogaert*, J. Peeters, J. Fostier, and F. Olyslager \\ Ghent University, Department of information technology (INTEC), \\ Belgium, http://www.intec.ugent.be
}

\section{Introduction}

The iterative solution of integral equations containing the Green function of the Helmholtz equation as the integration kernel requires repeated matrix-vector products. These products can be accelerated by means of a so-called fast multipole method (FMM). Of the many fast multipole methods in use today, the Multilevel Fast Multipole Algorithm (MLFMA) is arguably among the most successful ones. It allows the simulation of electrically large structures that are intractable with direct or unaccelerated iterative solvers. Testimony to the MLFMAs myriad uses is its implementation in various commercial EM software packages such as FEKO and CST Microwave studio. However, the MLFMA has one big drawback: a numerical instability prevents the method from being used on low frequency (LF) interactions, i.e. interactions between sources and observers that are less than approximately one wavelength apart. As a consequence configurations containing significant subwavelength geometrical detail cannot be efficiently treated using the MLFMA alone and a hybrid method is necessary. However, the LF methods in use today are generally less efficient due to non-diagonal translation operators (multipole methods) or the need for six radiation patterns (spectral methods). In this contribution a novel algorithm, called the Nondirective Stable Plane Wave Multilevel Fast Multipole Algorithm (NSPWMLFMA) [1], will be presented that is stable at LF, exhibits diagonal translation operators and requires only one radiation pattern. The method is based on an analytical expression for a translation operator in the $z$-direction. This translation operator is made numerically stable using a shift of the integration path into the complex plane. It even has a DC-limit. A QR-based method is then used to extend the applicability to all the other translation directions. The algorithm has also been parallelized using Open FMM [2]. Finally some numerical results will be shown.

\section{The LF breakdown of the MLFMA}

The LF breakdown can be understood by looking at the translation operator of the MLFMA

$$
T\left(k \boldsymbol{r}_{T}, \theta, \phi\right)=\sum_{l=0}^{L}(2 l+1) j^{-l} h_{l}^{(2)}\left(k r_{T}\right) P_{l}\left(\hat{\boldsymbol{k}}(\theta, \phi) \cdot \hat{\boldsymbol{r}}_{T}\right)
$$

with $k$ the wavenumber, $\boldsymbol{r}_{T}=r_{T} \hat{\boldsymbol{r}}_{T}$ the translation vector and $\hat{\boldsymbol{k}}(\theta, \phi)=\cos \phi \sin \theta \hat{\boldsymbol{x}}+$ $\sin \phi \sin \theta \hat{\boldsymbol{y}}+\cos \theta \hat{\boldsymbol{z}}$. The functions $P_{l}(\cdot)$ are the Legendre polynomials. Because the spherical Hankel function $h_{l}^{(2)}\left(k r_{T}\right)$ increases exponentially as a function of $l$ if $l>k r_{T}$, the terms with a low $l$ are eventually swamped in the high order terms. Obviously, the loss of the low order terms is catastrophic because they contribute the most to the addition theorem. 
Various approaches have been explored to deal with this problem. Maybe the most radical approach is to replace the MLFMA with a method based on the spectral representation of the Green function [3]. Another approach is to construct a hybrid method where the LF interactions are treated using a multipole [4] or spectral representation [5] based method. However, the multipole based method does not exhibit diagonal translation operators and the spectral methods require six radiation patterns for each box, making all these approaches less efficient than the MLFMA, if it were not numerically unstable. Indeed, it is stated in [5] that the high frequency technique (MLFMA) should be used whenever possible. Hence, a formulation of the MLFMA that is stable at LF is desirable. A first attempt to obtain such a formulation can be found in [6], where a complex shift of the integration path is performed and the translation operators are found numerically. However, the achievable accuracy is rather limited [7].

\section{A stable translation in the $z$-direction}

The method presented here is based on a uniform discretization of the addition theorem, as described in [8]. This means that the double Fourier spectrum of the translation operator times $|\sin \theta|$ has to be truncated at a bandwidth $L$. For a translation in the $z$-direction, the truncated translation operator $\tilde{T}\left(k r_{T} \hat{\boldsymbol{e}}_{z}, \theta, \phi\right)$ depends only on $\theta$ and can be written as follows

$$
\begin{gathered}
\tilde{T}\left(k r_{T} \hat{\boldsymbol{e}}_{z}, \theta, \phi\right)=\sum_{n=-L}^{L} b_{n} e^{j n \theta}, \\
b_{n}=\frac{1}{2 \pi} \sum_{l=0}^{L}(2 l+1) j^{-l} h_{l}^{(2)}\left(k r_{T}\right) f_{n}^{l} .
\end{gathered}
$$

The coefficients $f_{n}^{l}$ can be calculated analytically and have the crucial property that $f_{n}^{l}=0 \quad \forall-l<n<l$. Through a series of further manipulations and a shift of the integration path into the complex plane, this allows the construction of a numerically stable translation in the $z$-direction. The analytical expression for $f_{n}^{l}$ and an explicit formula for the magnitude of the complex shift can be found in [1].

\section{Stable translations in the other directions}

In order to obtain stable translations in a general direction $\boldsymbol{r}_{T}=r_{T} \overline{\mathbf{R}} \cdot \boldsymbol{e}_{z}$ with $\overline{\mathbf{R}}$ a $3 \times 3$ rotation matrix, it is necessary to express it as a translation in the $z$-direction

$$
h_{0}^{(2)}\left(k\left|\boldsymbol{r}_{A}+\boldsymbol{r}_{T}\right|\right)=\frac{1}{4 \pi} \int_{0}^{2 \pi} \int_{0}^{\pi} e^{-j(\overline{\mathbf{R}} \cdot \boldsymbol{k}) \cdot \boldsymbol{r}_{A}} T\left(k r_{T} \boldsymbol{e}_{z}, \theta, \phi\right) \sin \theta \mathrm{d} \theta \mathrm{d} \phi .
$$

The key problem is that the discretization points of the radiation pattern are rotated differently for each translation direction. Therefore we would need a separate set of plane waves for each different translation, which would be very inefficient. To avoid this, a QR is used to select the least dependent plane waves, such that they constitute a basis for the other plane waves. This process then allows the stable translation operators in the $z$-direction to be transformed such that they can be used on the selected plane waves. 


\section{Numerical results}

The accuracy of the proposed method was tested on the configuration shown in Figure 1 . The sides of the boxes are $1 \mathrm{~m}$ long. Figure 2 shows the obtained accuracy as a function of the frequency. The accuracy is defined as the maximum relative error of all the 64 interactions between the vertices of box 1 and box 2 . As can be seen,

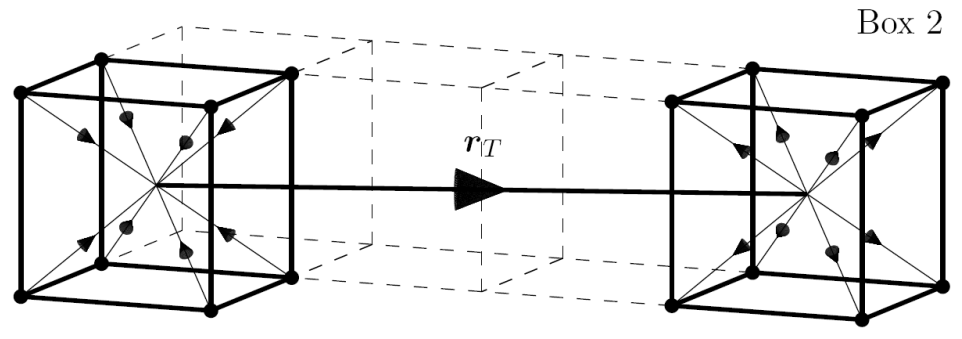

Box 1

Figure 1: The geometry for testing the accuracy.

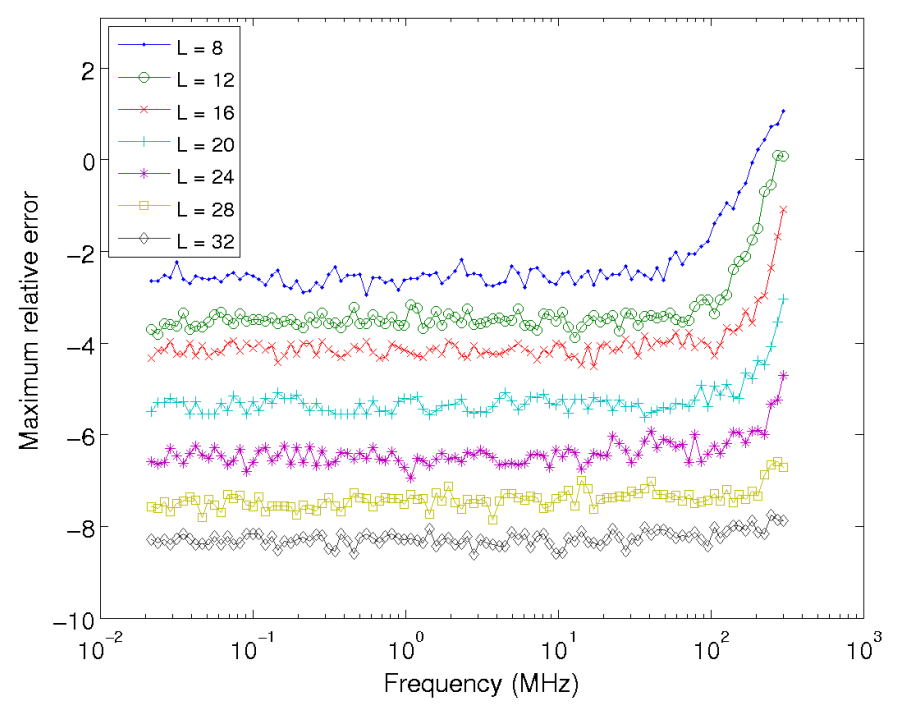

Figure 2: The maximum relative error as a function of the frequency for various truncation bounds $L$.

the method keeps on working fine even for very low frequencies. In fact the method remains stable all the way down to DC. This limit is derived in [1]. For high frequencies the error increases because the required $L$ for a certain accuracy increases. As for simulation results, Figure 3 shows simulations of a plane wave impinging on an A380 airplane. These simulations were performed using an asynchronously parallelized $[2,9]$ vectorial version of the hybrid MLFMA - NSPWMLFMA.

\section{References}

[1] I. Bogaert, J. Peeters, and F. Olyslager, "A nondirective plane wave MLFMA stable at low frequencies," Submitted for IEEE Trans. Ant. and Propagation. 


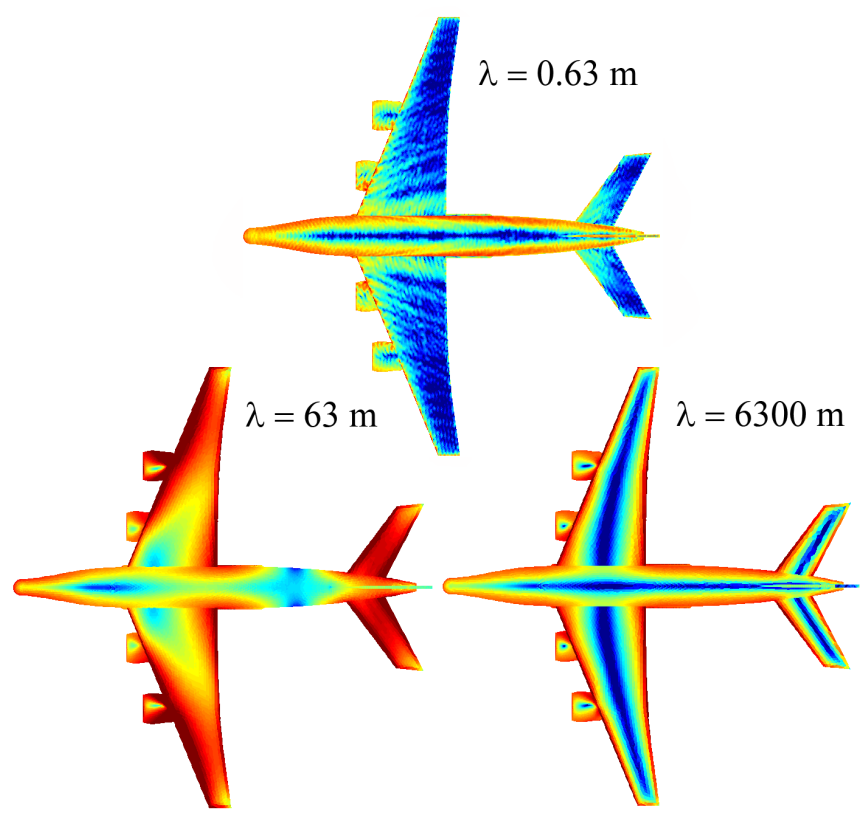

Figure 3: Currents on the A380 airplane for plane waves impinging from the left. The wavelengths are $0.63 \mathrm{~m}$ (top), 63m (bottom left) and $6300 \mathrm{~m}$ (bottom right) respectively. The number of unknowns is 500000 (top) and 117000 (bottom left and right).

[2] J. Fostier, J. Peeters, and F. Olyslager, "Open FMM." [Online]. Available: http://openfmm. intec.ugent. be

[3] E. Darve and P. Havé, "A fast multipole method for Maxwell equations stable at all frequencies," Philosophical Transactions of the Royal Society A, vol. 362, no. 1816, pp. 603-628, Mar 2004.

[4] L.J. Jiang and W.C. Chew, "A mixed-form fast multipole algorithm," IEEE Trans. Antennas Propag., vol. 53, no. 12, pp. 4145-4156, Dec 2005.

[5] H. Cheng et. al., "A wideband fast multipole method for the Helmholtz equation in three dimensions," J. Comput. Phys., no. 216, pp. 300-325, 2006.

[6] L. Xuan et. al., "A broadband multilevel fast multipole algorithm," in Proceedings of the IEEE AP-S International Symposium, Monterey, CA, vol. 2, pp. 1195-1198, June 2004.

[7] H. Wallen and J. Sarvas, "Translation procedures for broadband MLFMA," Progress In Electromagnetics Research, no. 55, pp. 47-78, 2005.

[8] J. Sarvas, "Performing interpolation and anterpolation entirely by fast Fourier transform in the 3-D multilevel fast multipole algorithm," SIAM Journal on Numerical Analysis, vol. 41, no. 6, pp. 2180-2196, 2003.

[9] J. Fostier and F. Olyslager, "An asynchronous parallel MLFMA for scattering at multiple dielectric objects," Accepted for IEEE Trans. Antennas Propag. 


\section{IEEE AP-S International Symposium and \\ USNC/URSI National Radio Science Meeting}

Copyright and Reprint Permission: Abstracting is permitted with credit to the source. Libraries are permitted to photocopy beyond the limit of U.S. copyright law for private use of patrons those articles in this volume that carry a code at the bottom of the first page, provided the per-copy fee indicated in the code is paid through the Copyright Clearance Center, 222 Rosewood Drive, Danvers, MA 01923. For other copying, reprint, or replication permission, write to IEEE Copyrights Manager, IEEE Operations Center, 445 Hoes Lane, P.O. Box 1331, Piscataway, NJ, 08855-1331. All rights reserved. Copyright (c) 2008 by the Institute of Electrical and Electronics Engineers, Inc. Personal use of this material is permitted. However, permission to reprint/republish this material for advertising or promotional purposes or for creating new collective works for sale or distribution to servers or lists, or to reuse any copyrighted component of this work in other works must be obtained from the IEEE.

\section{Preliminary Version}

Support: If you have problems or questions related to the installation of this disc, please contact the 3WAIsmen at FAX: (818) 952-0183 or e-mail: wais3men@yahoo.com

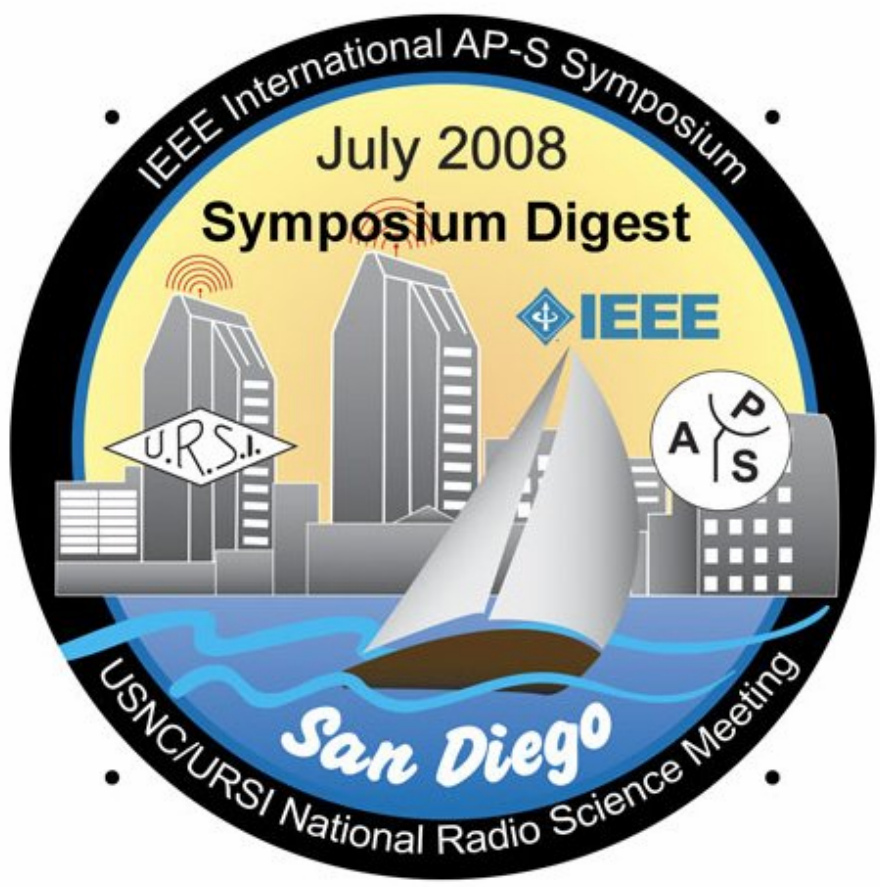

July 5-12, 2008 WAIIS $\overline{\mathbf{Z}}^{\text {mnnem }}$
San Diego, CA

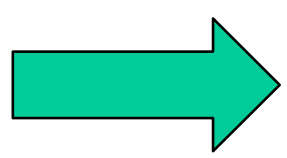

